\title{
Plataforma Robótica para Busca de Pessoas em Locais de Risco
}

\author{
Edgar Natanael de Vasconcelos Gregório ${ }^{1}$ \\ Ricardo Teixeira da Silva ${ }^{1}$ \\ ${ }^{1}$ Escola Politécnica de Pernambuco, Universidade de Pernambuco, Recife, Brasil \\ E-mail do autor principal: Edgar Natanael de Vasconcelos Gregório edgarnatanael28@hotmail.com
}

\section{Resumo}

Este trabalho foi escrito e organizado com o objetivo de dar suporte à localização de pessoas em locais de risco. A sua criação se deu na necessidade de realizar, busca de pessoas em locais de difícil acesso ao corpo de bombeiros e onde o terreno traga riscos iminente para esses profissionais. Robôs já são usados para auxiliar os profissionais de segurança publica em diversas áreas do Brasil, contando com fortes estruturas e controladas remotamente, possuindo câmeras com altas definições onde podem visualizar vítimas em meios de entulhos ou em locais inapropriados de difícil acesso as equipes de resgates, com tecnologias capazes de realizar o serviço de forma a reduzir gastos, tempo e com a maior eficiência possível. O robô desenvolvido possui acessórios importantes para a realização do trabalho de busca, usando tecnologias que podem ajudar a salvar vidas, obtendo uma boa visualização do local e do interior do acidente de forma nítida através de uma câmera IP que transmite a imagem através do WI-FI numa distância de aproximadamente 50 metros em locais fechados. A câmera possui infravermelho para realizar imagens onde a iluminação é precária. $A$ locomoção do robô é através de uma plataforma de tanque chassi robô com esteiras, aumentando sua aderência e oferecendo uma maior estabilidade no seu deslocamento. A plataforma possui amortecedores para dar suavidade durante o seu deslocamento. A sua comunicação é feita através do módulo ESP8266, que realiza a transmissão de dados através do WI-FI, chegando numa distância de aproximadamente de 90 metros em locais abertos e 50 metros aproximadamente em locais fechados. Tendo em vista que o robô é apenas um protótipo, várias outras implementações podem ser realizadas, adicionando outras funcionalidades.

Palavras-Chave: Bombeiro Militar; Vítimas; Robôs.

\section{Abstract}

This work was written and organized with the purpose of supporting the location of people in places of risk. Its creation was in need of carrying out, search of people in places of difficult access to the fire brigade and where the terrain poses imminent risks for these 
professionals. Robots are already used to assist public safety professionals in diverse areas of Brazil, with strong structures and remotely controlled, having cameras with high definitions where they can visualize victims in debris means or in inappropriate places of difficult access to rescue teams, with technologies capable of performing the service in order to reduce expenses, time and with the highest possible efficiency. The developed robot has important accessories to carry out the search, using technologies that can help save lives, getting a good view of the site and the interior of the accident in a clear way through an IP camera that transmits the image through the WI- FI at a distance of approximately 50 meters indoors. The camera has infrared for images where illumination is poor. The locomotion of the robot is through a robot chassis tank platform with mats, increasing its grip and offering greater stability in its displacement. The platform has shock absorbers to give smoothness during its displacement. Its communication is made through the module ESP8266, which transmits data through the WI-FI, arriving at a distance of approximately 90 meters in open places and 50 meters approximately in closed places. Since the robot is only a prototype, several other implementations can be performed, adding other functionalities.

Key-words: Firefighter Military; Casualties; Robots. 


\section{Introdução}

O corpo de Bombeiro Militar do estado de Pernambuco e de todo Brasil tem sua garantia legal na CONSTITUIÇÃO DA REPÚBLICA FEDERATIVA DO BRASIL de 1988, no Art. 144 [1].

O corpo de Bombeiro Militar de Pernambuco é uma instituição que faz parte das forças de segurança do estado sendo responsáveis pelo combate a incêndios, pela preservação do patrimônio ameaçado de destruição, pelo resgate de vítimas de incêndios, afogamentos, acidentes ou catástrofes e pela conscientização da população sobre medidas de segurança contra incêndios [2].

Os profissionais atuam em diversas situações de desastres e catástrofes e estão aptos a realizar qualquer salvamento e cumprir a sua missão, mesmo com o risco da sua própria vida e esse é um fator bastante preocupante, pelo fato dos mesmos participarem diretamente das operações de salvamento e busca.

O uso de robôs em operações de Bombeiros tem sido cada vez mais estudado devido à exposição destes profissionais a graves riscos em salvamentos. São diversos os tipos de robôs usados, como robôs extintores e cortadores, que podem ser utilizados para auxiliar salvamentos [3].

Em várias partes do Brasil, as empresas têm buscado o aumento da eficiência e na prestação de serviço por meio da utilização da tecnologia, para o planejamento tático e estratégico, bem como para formulação de indicadores sobre os seus resultados, sendo um apoio na modernização, desenvolvendo assim novas possibilidades para uma mudança radical e de novos paradigmas [4].

O protótipo robótico desenvolvido tem como objetivo final ser usado em operações de busca por vítimas de acidente em locais que tragam risco iminente aos profissionais, revelando uma gama de informações do interior do local como: o tipo do terreno, extração de imagens, posição dos entulhos, posição das vítimas e a sua condição clinica médica, com nitidez e em tempo real.

O robô será controlado através de um computador remoto (notebook), o qual enviará os comandos para a plataforma robótica através do sinal de WI-FI de um roteador para o dispositivo wireless ESP8266 conectado na plataforma robótica, permitindo uma conexão entre os dispositivos sem a utilização de fios.

O mecanismo robótico foi construído para se locomover através de uma plataforma de Robô Tanque de choque crawle, onde seus movimentos serão realizados através de esteiras dando uma maior aderência no seu deslocamento.

Para obter uma iluminação e consequentemente uma boa visualização do local onde o robô estará inserido um sensor de luminosidade ligado a um microcontrolador acionará uma lanterna do tipo cree led trazendo iluminação ao local.

O computador estará com o profissional de resgate onde poderá visualizar em tempo real todas as informações possíveis do interior do local da ocorrência, através da Câmera IP, que estará acoplada a plataforma robótica, podendo assim tomar as devidas decisões e precauções antes de entrar no local.

\section{Trabalhos Relacionados}

Por varias partes do Brasil e no mundo os governos estão cada vez mais investindo em maquinas para busca e salvamento de vítimas de desastres. Um exemplo disso é o Japão onde BL Autotec desenvolveu um robô para a realização de busca e resgate com uma câmera digital para operar em lugares escuros e com um braço mecânico ele consegue retirar materiais perigosos e pesados, como escombros e outros [5].

O Centro de Pesquisa Assistida por Robô e Salvamento (CRASAR) do Texas, nos Estados Unidos da América, tem como sua principal missão realizar o aperfeiçoamento e a prevenção de desastres e salvamento de pessoas usando robôs com tecnologias avançadas.

O CRASAR tem uma grande participação do uso de seus robôs nos maiores desastres ao redor do mundo.

O CRASAR realiza pesquisas para explorar o desenvolvimento de novas tecnologias na área de robótica e sistemas não tripulados para fins de salvamento e resgate de humanos e animais em perigo de vida, possuindo assim o maior numero de robôs de resgate que atua em qualquer área, seja: terra, mar ou ar [6].

De acordo com essas empresas a utilização de maquinas inteligentes para o uso e o bem estar do ser humano tem um potencial de crescimento ao longo dos anos.

O protótipo proposto neste artigo pode ser enviado com o objetivo de encontrar pessoas, nas mais variadas situações e locais, onde ocorreu o acidente, podendo assim, entrar em terrenos como: entulhos, escombros, terra ou areia.

http://dx.doi.org/10.25286/repa.v2i2.550 
O robô tem a facilidade de comunicação pelo fato da conexão ser por meio de WI-FI, com o seu controle, podendo entrar no ambiente de tal forma que o seu controlador esteja fora do local com uma distância segura.

A tecnologia usada na sua construção apenas permitirá que ele seja usado em desastre que tenham locais de áreas secas e de temperatura no máximo de $90^{\circ} \mathrm{C}$ e no mínimo de $-40^{\circ} \mathrm{C}$.

Com o uso dessa tecnologia podemos ter uma participação ampla e construção de outras maquinas para o auxilio humano no sentido de aperfeiçoar as buscas nos resgates de forma segura e eficiente.

\section{Desenvolvimento}

O protótipo foi desenvolvido com a junção da vários módulos eletrônicos e dois microcontroladores.

O robô é formado por um Chassi Tanque de Choque Crawle, um microcontrolador PIC 16F877A e um 18F2550, uma câmera IP, lanterna tática Cree LED, um sensor de luminosidade LDR, módulo wireless ESP8266, linguagem de marcação HTML para a criação da página web e a módula ponte-H L298N.

Para o desenvolvimento dos códigos foram usadas duas plataformas:

- Para o Pic o 'compiler ccs';

- Para o ESP8266 'arduino'.

\subsection{Dispositivos}

\subsubsection{Chassi Tanque de Choque Crawle}

Para a locomoção e base do projeto foi escolhido um chassi com duas esteiras controladas por motores independentes, construído com o material de plástico, com amortecedores nas rodas e esteira de borracha para garantir uma forte aderência adversos tipos de terreno [7].

A utilização de um chassi Tanque com esteira foi escolhida pelo fato do mesmo possuir uma grande estabilidade e um maior contato com o solo, diminuindo a patinagem no solo e até em terrenos que haja inclinações [8].

As principais características são:

- Alimentação: 3VCC - 9VCC;

- Massa: 550g;

- Largura de Faixa: $2.3 \mathrm{~cm}$ feito de borracha;

- Tamanho: $30 \mathrm{~cm} \times 15 \mathrm{~cm} \times 7.6 \mathrm{~cm}$.
A Figura 1 apresenta a foto do chassi utilizado neste projeto.

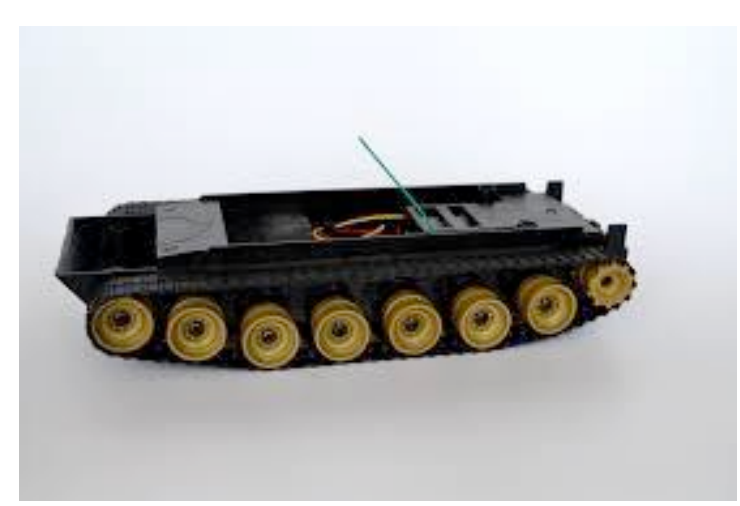

Figura 1: Chassi tanque de choque crawle.

\subsubsection{Conhecendo a Plataforma do PICmicro}

O Microcontrolador utilizado para o controle do projeto é o PIC 16F877A.

O PIC 16F877A é um microcontrolador da família 16, é amplamente utilizado em varias aplicações acadêmicas e comerciais, são bastante utilizados por causa de seu baixo custo no mercado eletrônico, possui uma grande e variada gama de aplicações em diversas áreas, possui uma enorme qualidade $\mathrm{e}$ disponibilidade no mercado.

É ideal para aplicações tais como: controle de máquinas, dispositivos robóticos, e assim por diante [9].

Dentro de alguns PICs conhecidos, o 16F877A possui as características suficientes para a construção deste projeto, como mostra a Figura 2. Entre suas características principais podemos citar:

- Microcontrolador de 40 pinos;

- 33 pinos configuráveis como entrada ou saída;

- 15 interrupções disponíveis;

- Memória de programação FLASH;

- Memória EEPROM (não volátil) interna com 256 bytes;

- Memória RAM com 368 bytes;

- Três timers (2x8bits e $1 \times 16$ bits);

- Comunicações seriais: SPI, $I^{2} C$ e USART;

- Conversores analógicos de 10 bits;

- Dois módulos CCP: Capture, Compare e PWM.

O Microcontrolador utilizado para o controle da iluminação é o PIC 18F2550, como mostra a Figura 3. 


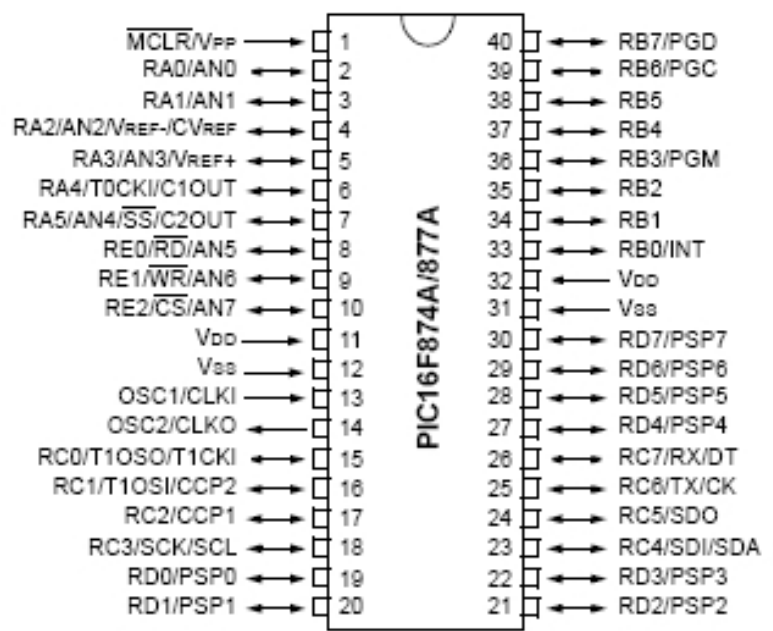

Figura 2: Pic 16F877A.

Figura 3: Pic 18F2550.

O microcontrolador PIC18F2550 é da família de microcontroladores de 8 bits e núcleo de 14 bits criada pela MICROCHIP. Ele permite controlar desde mais simples programa até um controle de um robô [10].

Dados Técnicos:

- Tensão máxima: $5.5 \mathrm{~V}$;

- Entradas e saídas digitais: 33;

- Corrente de saída: 25 mA;

- Memória Flash: 14.3 kB;

- Memória SRAM: 2048 bytes;

- Memória EEPROM: 256 bytes;

- Velocidade do clock: $0-20 \mathrm{MHz}$.

\subsubsection{Câmera IP}

A câmera IP referenciada na Figura 4 foi desenvolvida para realizar a visualização de imagens

em tempo real de qualquer lugar através do uso da internet.

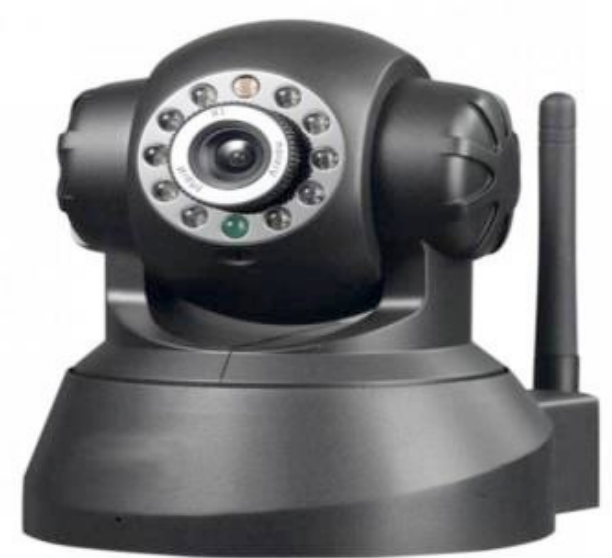

Figura 4: Câmera IP.

Possui um motor que possibilita sua movimentação horizontal em $270^{\circ}$ e vertical $90^{\circ}$, onde pode ser controlada de qualquer lugar através do computador ou celular ligada à rede de internet através do próprio programa.

Possui um microfone e um autofalante o qual permite que você possa conversar com vítimas no local de um possível acidente, através do seu computador ou celular.

Possui uma lâmpada infravermelha que é rapidamente acionada de modo automático conforme a luminosidade do local, podendo assim realizar a filmagem no período noturno.

A câmera pode ser acionada estando conectada a um fio de internet $\mathrm{Rj} 45$ ou simplesmente pode ser acionada através de um roteador [11].

\subsubsection{Módulo WI-FI ESP8266 ESP- 01}

O modulo wireless ESP8266, apresentado na Figura 5, foi criado para realizar a conexão de microcontroladores com a uma conexão WI-FI de uma forma bem simples e fácil.

Este módulo pode ser usado de duas formas, como um ponto de acesso (Access Point) ou como uma estação (Station), para poder realizar o envio ou o recebimento dos dados.

http: / /dx.doi.org/10.25286/repa.v2i2.550 
A comunicação deste módulo com os microcontroladores é feita utilizando uma interface serial e o seu firmware (conjunto de instrução operacional), podendo ser atualizado constantemente assim que necessário [12].

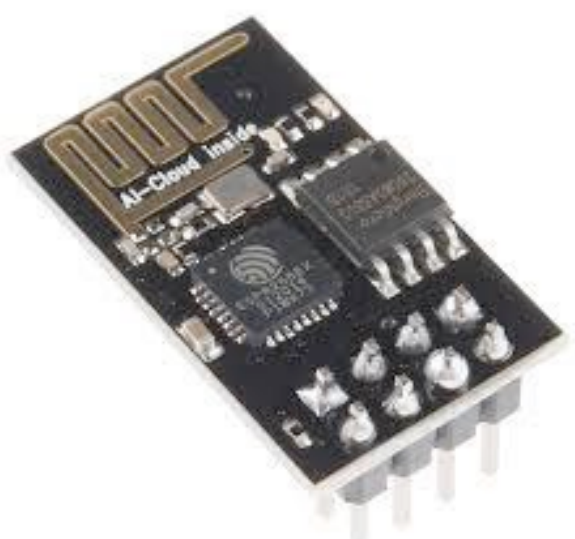

Figura 5: Módulo WI-FI ESP8266 ESP-01.

O ESP8266 possui uma antena embutida para a transmissão dos dados.

- Conexão a redes padrão 802.11 B/G/N;

- Alcance aproximado: 91 metros;

- Tensão de operação: 3.3 VDC;

- Comunicação serial: pinos TX e RX;

- Modos de operação: Cliente, Access Point, Client+AccessPoint;

- Modos de segurança wireless OPEN/WEP/WPA_PSK/WPA2_PSK/WPA_WPA2 _PSK;

- Suporta comunicação TCP e UDP, com até cinco conexões simultâneas.

\subsubsection{Sensor de Luminosidade LDR}

O LDR (Light Dependent Resistor) significa resistor dependente de luz, é um componente em que a sua resistência varia de acordo com a luminosidade do local, ou seja, quanto, mais luz no ambiente em que está o sensor menor será a sua resistência.

Este sensor de luminosidade mostrado na Figura 6 , pode ser utilizado em vários projetos com todos os tipos de microcontroladores [13].

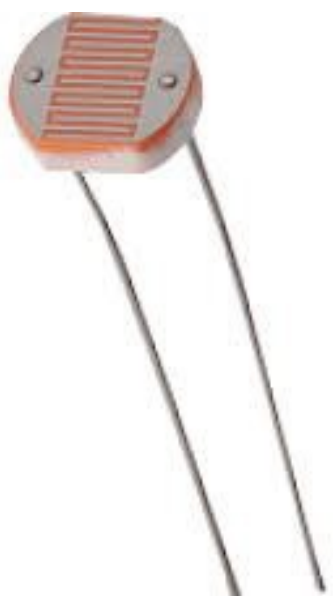

Figura 6: Foto do sensor de luminosidade LDR.

\subsubsection{Módulo L298N}

O módulo é baseado no circuito integrado L298N que consiste em uma ponte-h e é bastante utilizado na área de robótica para o controle de motores. 0 modulo tem saída para controle de dois motores DC como mostra a Figura 7.

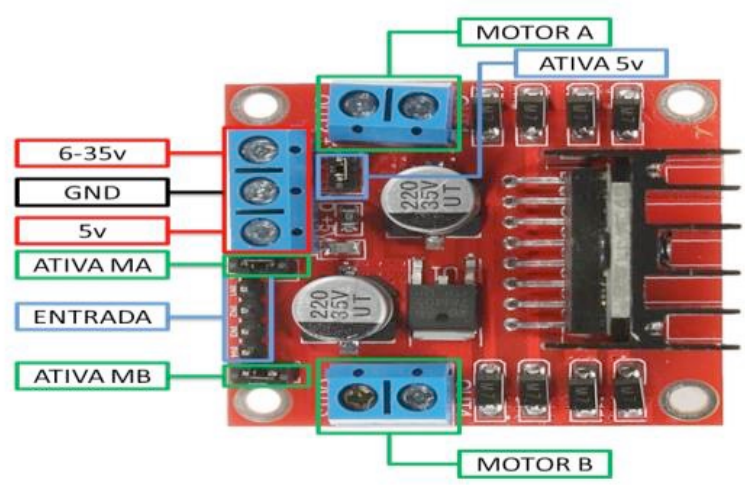

Figura 7: Módulo ponte H L298N.

O módulo ainda permite o controle da velocidade e da direção dos motores.

O módulo possui 6(seis), pinos de entrada, como também 4 (quatro), pinos de saída e alimentação para a placa numa tensão de $5 \mathrm{VCC}$ e alimentação para os pinos que alimentam o motor de 6VCC até 35VCC [14].

Características principais:

- Controle de 2 motores DC ou 1 motor de passo;

- Corrent de máxima: 2 A por canal ou 4A; 
- Tensão lógica: $5 \mathrm{~V}$;

- Corrente lógica: 0 36 mA;

- Limites de temperatura: -20 a $+135^{\circ} \mathrm{C}$;

- Potência máxima: $25 \mathrm{~W}$.

\subsubsection{Fonte de Alimentação}

A bateria utilizada na alimentação do protótipo foi a do tipo Lithium-Polymer (LIPO) representada na Figura 8. É uma bateria que tem seus eletrólitos de sais de lítio retidos em um polímero sólido como o oxido de polietileno [15].

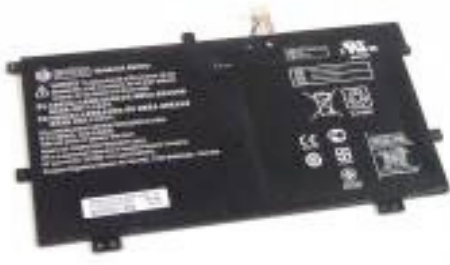

Figura 8: Bateria LIPO.

A opção pelo o uso deste tipo de bateria é que possui uma grande capacidade de armazenar energia, comparadas com outras baterias.

A bateria Lipo usada é constituída de duas células (2S), cada célula armazena $3.7 \mathrm{~V}$ logo o seu armazenamento total é de $7.4 \mathrm{~V}$, pois estão ligadas em série. A carga é de $2950 \mathrm{mAh}$ e $15 \mathrm{C}$ o que representa uma descarga de 15A.

Características principais:

- Números de Células: 2;

- Voltagem: 7.4V;

- Capacidade mAh: 2950.

\subsubsection{Lanterna Led Cree}

A lanterna cree led referenciada na Figura 9, atinge até $50 \mathrm{~m}$ com um bom foco de nitidez, bastante usada no mercado [16].

Principais características:

- Modelo: Lanterna CREE LED.

- Potência: 3 w.

- Cor: Preta.

- Marca: CREE LED.

- Alimentação: 3x baterias AAA.
- Ilumina até 50 metros de distância com muita nitidez.

- Peso: 91 gramas.

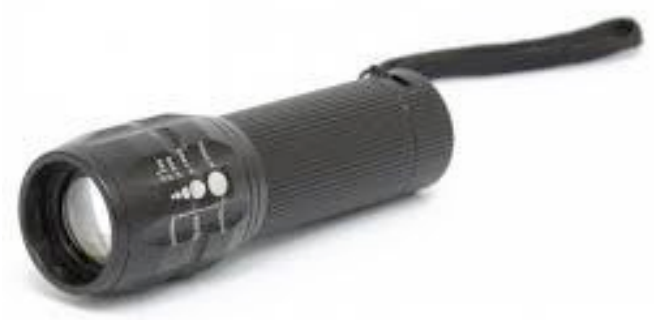

Figura 9: Lanterna Cree Led.

\section{Construção}

O desenvolvimento e a construção deste projeto foram realizados em 06(seis) etapas:

- ESP8266 ESP-01:

Foi desenvolvido o código de linguagem em ' $C$ ', para o modulo, usando a IDE de desenvolvimento do "arduino".

Junto à linguagem foi criado um layout de comunicação do modulo com o notebook, em HTML.

No HTML foram criados 7 (sete), botões:
1. Ligar;
2. Desligar;
3. Frente;
4. Ré;
5. Direita;
6. Esquerda;
7. Parar.

Após a criação da linguagem foi realizado a gravação no modulo através de um conversor TTL - USB.

As informações serão transmitidas pelas portas seriais do modulo RX e TX.

- Desenvolvimento da programação em C, para o Microcontrolador PIC 16F877A e o PIC 18F2550:

O microcontrolador 16F877A receberá pelas portas serias TX e RX, pino 25 e pino 26 respectivamente as informações vindas do 
modulo ESP8266 ESP - 01, e assim repassado para a porta B (b0, b1, b2, b3, b4 e b5).

Esses pinos ativaram as entradas do modulo ponte $\mathrm{H}$ L298N.

O microcontrolador $18 \mathrm{~F} 2550$ receberá as informações vinda do sensor de luminosidade LDR, e acionará a porta B (b0). Um circuito integrado 7408 (porta lógica AND) receberá o sinal do pic, acendendo assim a lanterna.

- Simulação no Proteus (Labcenter Electronics):

Foi realizada a simulação do Pic 16F877A no proteus, com a comunicação serial, juntamente com módulo ponte H L298N. O Pic $18 F 2550$ também foi testado com o sensor LDR.

- Modulo L298N:

Próximo passo foi à conexão do Pic 16F877A com o modulo L298N e consequentemente com os motores DC da plataforma robótica.

- Código da linguagem HTML:

Para a obtenção dos dados vindo do ESP8266 para a página web no notebook, para uma melhor visualização do controle.

\section{- Câmera IP:}

A transmissão da imagem será realizada diretamente para o notebook através do WI-FI mostrando uma boa nitidez do local, com um ambiente iluminado ou sem iluminação.

\section{- Iluminação:}

Para a iluminação do robô foi usado o microcontrolador Pic $18 \mathrm{~F} 2550$ conectado ao sensor LDR, onde ao detectar abaixa iluminação do local o Pic acionará a lâmpada.

A Figura 10 mostra a interligação entre os módulos eletrônicos e o microcontrolador Pic 16F877A, para a locomoção da plataforma chassi tanque de esteiras.

A Figura 11 mostra a interligação entre o sensor, pic $18 F 2550$ e a lanterna cree led, formando o farol do protótipo robótico.

A Figura 12 mostra a comunicação da câmera IP com o notebook.

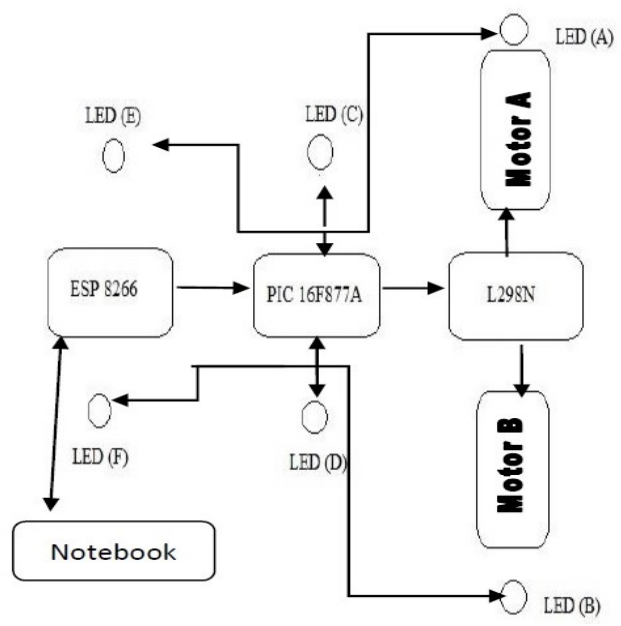

Figura 10: Diagrama de blocos I do projeto.

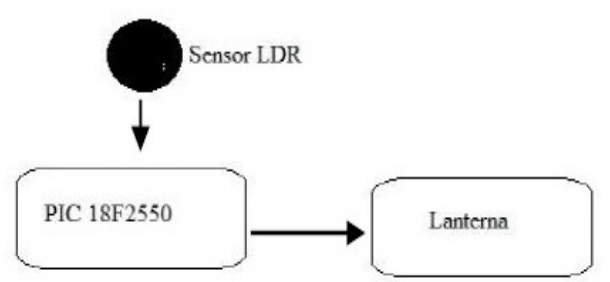

Figura 11: Diagrama de blocos II do projeto.

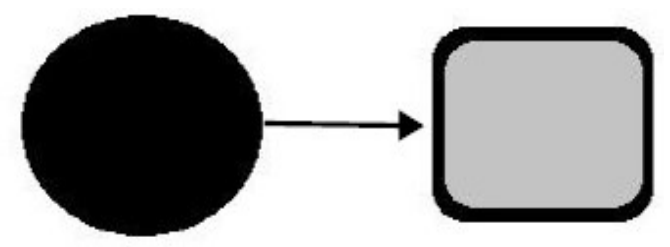

Câmera IP

Notebook

Figura 12: Diagrama de blocos III do projeto

\subsection{Fluxograma do Código}

O fluxograma do código desenvolvido é mostrado na Figura 13 e Figura 14. O código foi desenvolvido em linguagem C, para o módulo ESP8266 e para os microcontroladores pic's.

- Comunicação do movimento da plataforma: 


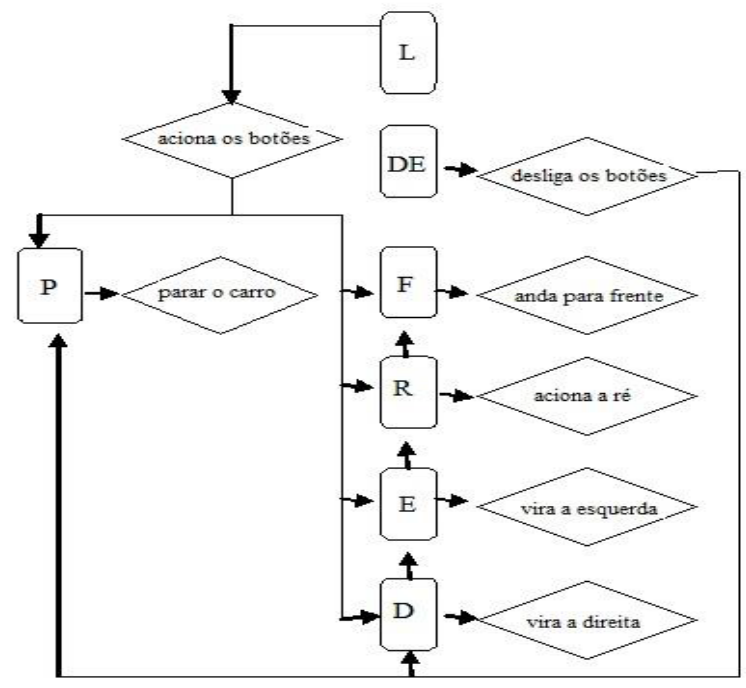

Figura 13: Fluxograma do código.

- Acionamento do farol:

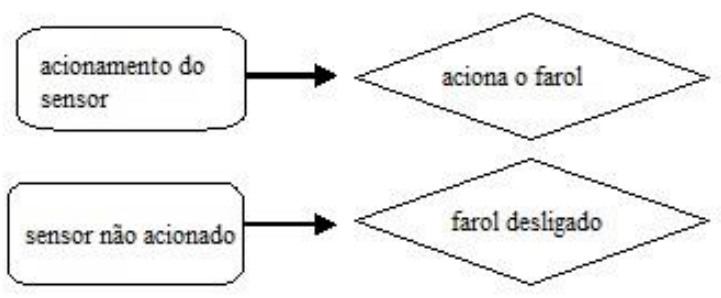

Figura 14: Fluxograma do código.

\subsection{Circuitos Elétricos}

A Figura 15 mostra o circuito eletrônico da locomoção do protótipo desenvolvido para o projeto.

A Figura 16 mostra o circuito eletrônico do farol do protótipo desenvolvido para o projeto.

\section{Teste}

Foram realizados diversos testes sobre 0 funcionamento do projeto, verificando sua utilidade no auxílio de busca e salvamento de pessoas em locais de riscos.

Foram realizados testes de:
- Controle;

- Tempo e consumo da bateria;

- Obtenção da imagem.

- Locomoção;

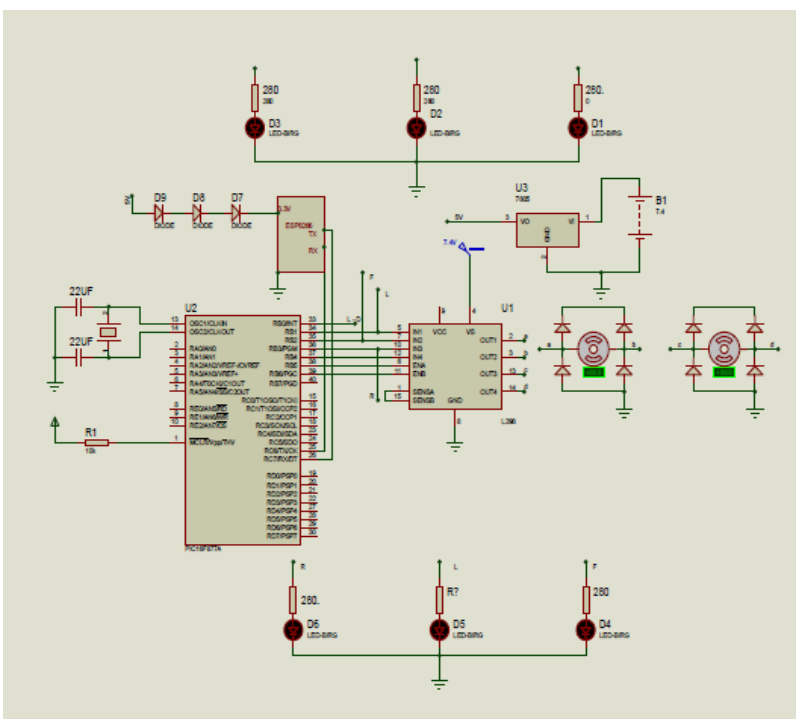

Figura 15: Circuito eletrônico da locomoção.

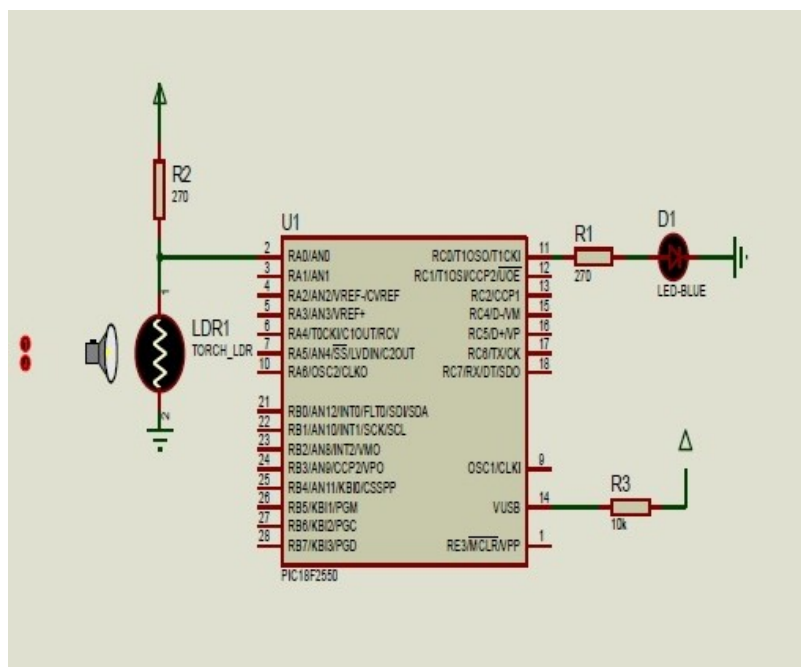

Figura 16: Circuito eletrônico do farol.

\subsection{O Controle:}

O controle de comunicação entre o computador e a plataforma robótica usando o ESP8266 ESP-01, funcionou com sucesso. A transmissão dos dados foi

http: //dx.doi.org/10.25286/repa.v2i2.550 
realizada em uma distancia de $90 \mathrm{~m}$ aproximadamente em locais abertos e $50 \mathrm{~m}$ aproximadamente em locais fechados.

Este alcance de sinal é determinado pelo o sinal do roteador, no caso usamos o TP-Link TL - WR740N, onde o seu alcance em metros é de $150 \mathrm{~m}$, em locais abertos.

A Figura 17 demonstra o controle da plataforma robótica acessada pelo notebook.

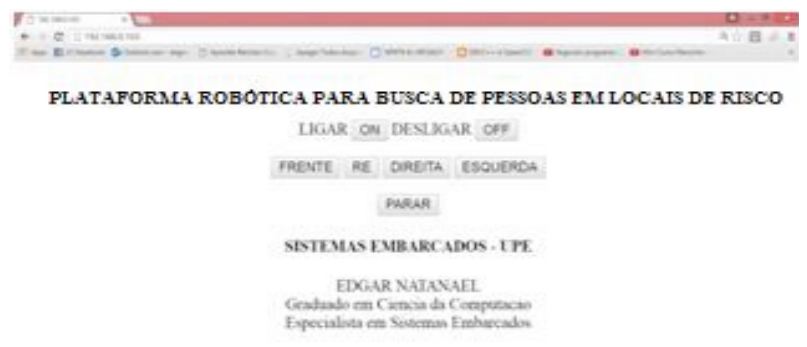

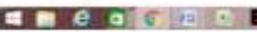

Figura 17: Circuito eletrônico da locomoção.

A Figura 18 demonstra o controle da plataforma robótica acessada pelo celular.

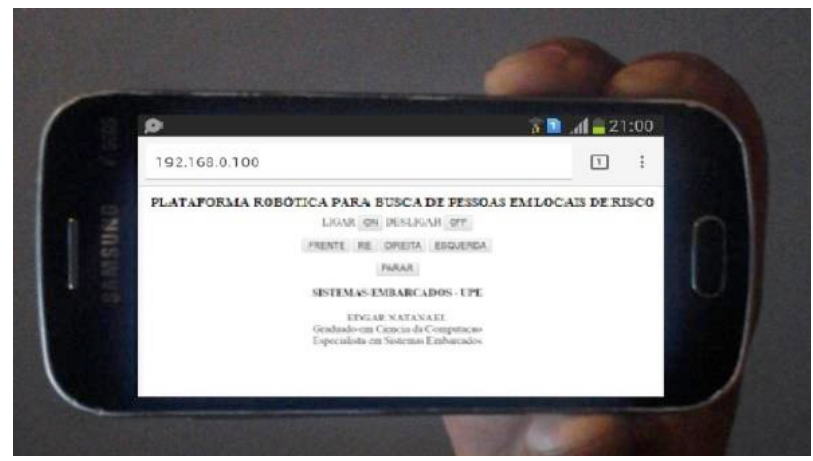

Figura 18: Comunicação do ESP8266 com o celular.

A Figura 19 mostra o programa de controle da câmera IP.

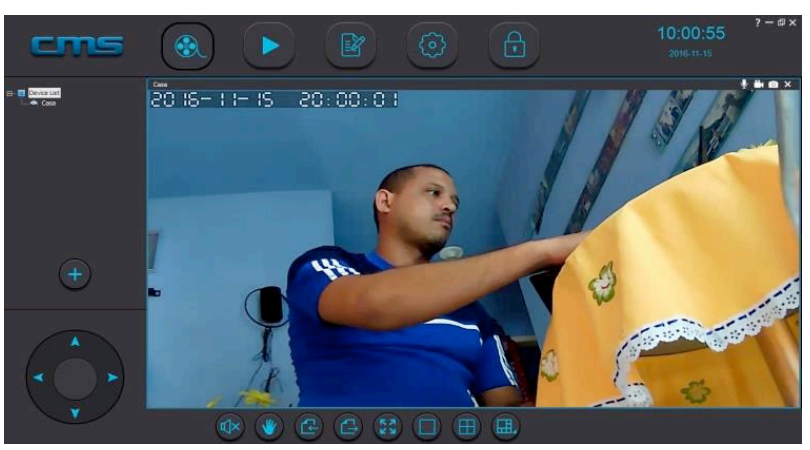

Figura 19: Ambiente do controle da câmera IP.

\subsection{Bateria:}

A bateria usada no robô como já foi especificada em cima é a do tipo LIPO e sua tensão é de $7.4 \mathrm{v}$ e $2950 m A$ h. O robô consome uma corrente mínima para o seu funcionamento de $1,1 \mathrm{~A}$ ou $1100 \mathrm{~mA}$. Com a bateria usada e o consumo do circuito o tempo de funcionamento do robô é de $2 \mathrm{~h} 41 \mathrm{~min}$ aproximadamente.

De acordo com algumas pesquisas foi encontrada uma fórmula usada por estudantes e profissionais para calcular o tempo de duração de uma bateria [17].

Tempo = Capacidade da bateria / Consumo do dispositivo.

Tempo $=2950 \mathrm{mAh} / 1100 \mathrm{~mA}$.

Tempo $=2 \mathrm{~h} 41 \mathrm{~min}$.

\subsection{Obtenção de Imagem:}

As imagens obtidas foram de uma boa qualidade de acordo com as especificações do fabricante da câmera IP e meramente ilustrativa, apenas para demonstrar a sua qualidade.

Principais características do programa da câmera (CMS):

- Botões de direções (Esquerda, Direita, Cima e para Baixo);

- Botão de alto falante;

- Botão de para fotos;

- Botão de filmagens.

A filmagem e as fotos da câmera são armazenadas em um cartão de memória acoplado na própria câmera. 
A câmera pode ser acessada pelo cabo $\mathrm{Rj} 45$ ou programada para ser acessada pela WI FI, no caso do nosso projeto. O alcance do acesso da câmera é determinado pelo roteador, no caso usamos o TP-Link TL - WR740N, onde o seu alcance em metros é de $150 \mathrm{~m}$, em locais abertos.

Em locais fechados constatamos que o alcance do sinal foi aproximadamente de $50 \mathrm{~m}$.

Ainda foi possível realizar a comunicação com o robô através do microfone e altofalante da câmera. 0 seu funcionamento é realizado da seguinte forma: para falar é pressionado o botão de voz e para ouvir ele é solto. A distância de alcance obedece ao mesmo critério que o sinal de WI-FI.

As Figuras a seguir demonstram a visão do controlador pelo notebook através da câmera IP, em ambiente com luz, com baixa luminosidade (infravermelho da câmera) e com o farol do robô.

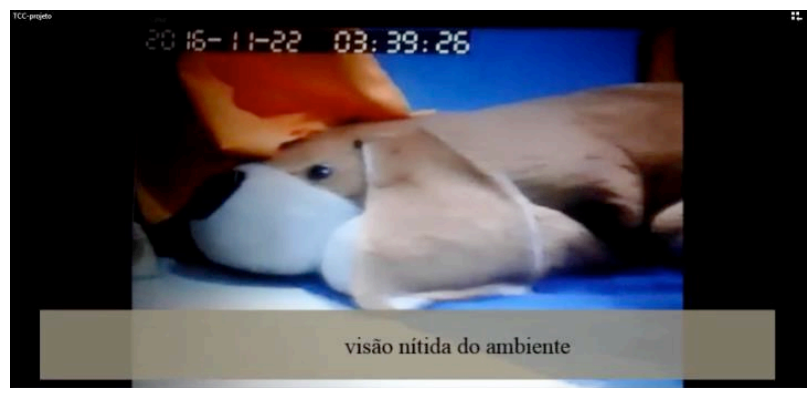

Figura 20: Ambiente com luz.

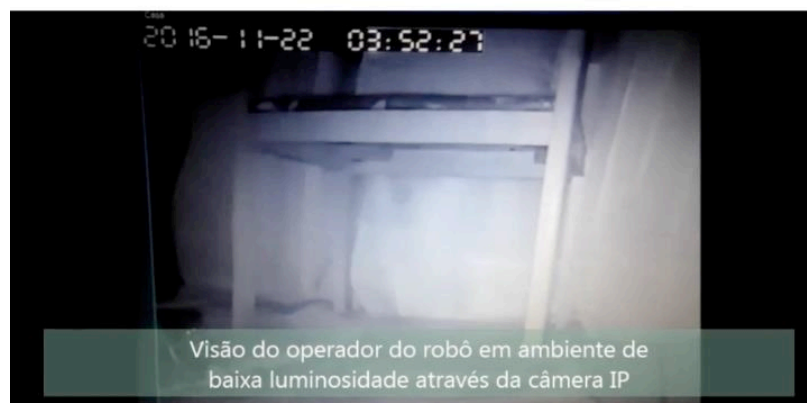

Figura 21: Ausência de luz (infravermelho).

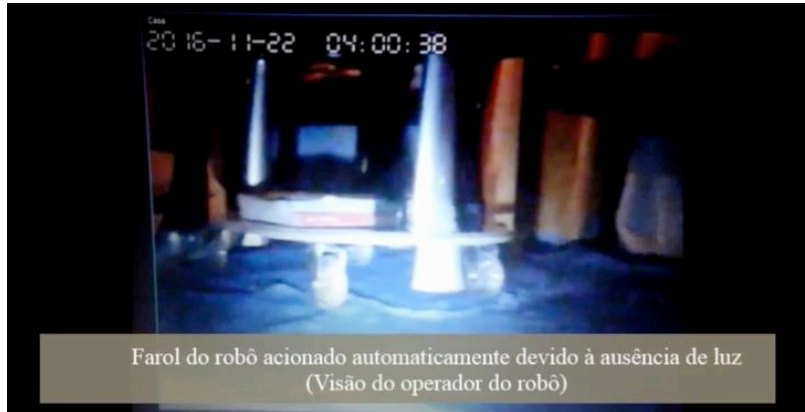

Figura 22: Farol da plataforma robótica ligada.

\subsection{Locomoção:}

Os terrenos onde foram realizados os testes da plataforma robótica, são do tipo arenoso (areia), com entulhos (resíduo da construção civil) e com alguns tipos de revestimento de pisos, como cimento e cerâmicas, obtendo assim um ótimo desempenho e uma estabilidade no seu deslocamento.

A Figura 23 mostra a plataforma robótica em terreno com entulhos.

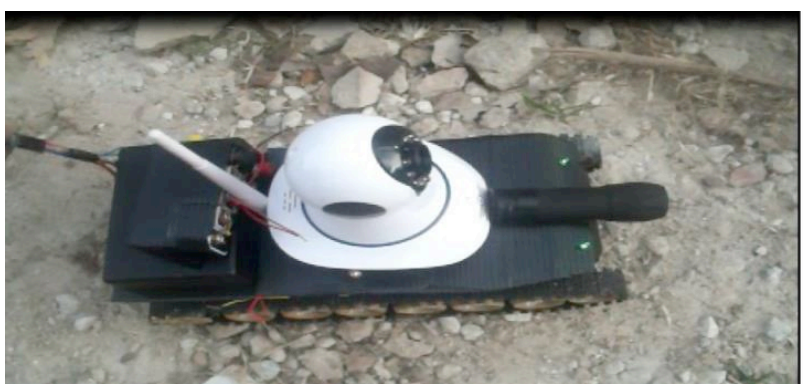

Figura 23: Terreno com entulhos.

A Figura 24 mostra a plataforma robótica em piso de cerâmica.

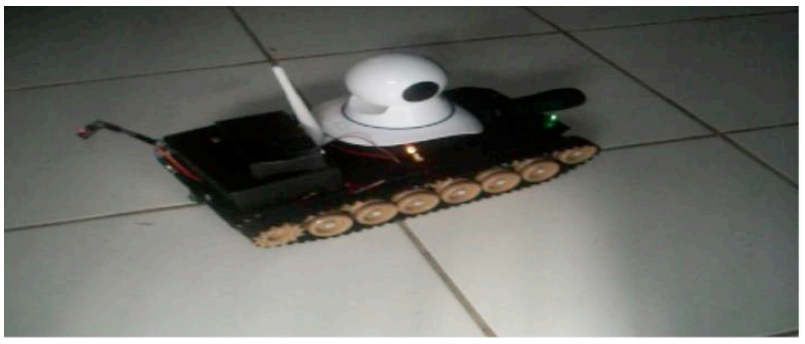

http://dx.doi.org/10.25286/repa.v2i2.550 
Figura 24: Piso de cerâmica.

A Figura 25 mostra a plataforma robótica em piso de cimento.

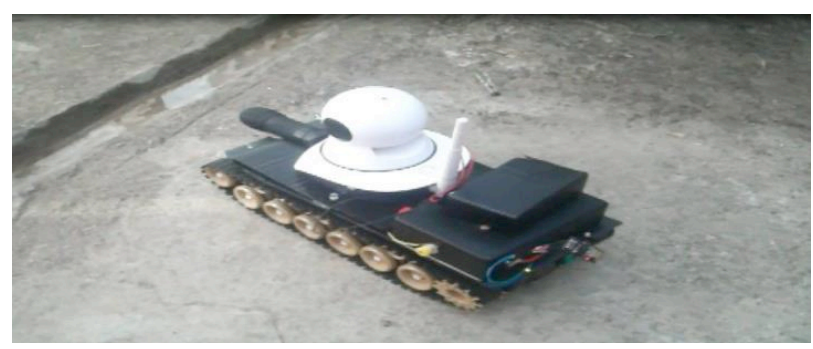

Figura 25: Piso de cimento.

\subsection{Iluminação:}

O sensor de Iluminação reagiu muito bem, com bastante precisão trazendo iluminação necessária quando o robô adentrou em local onde iluminação era precária, como mostra as Figuras 26 e Figuras 27 seguir.

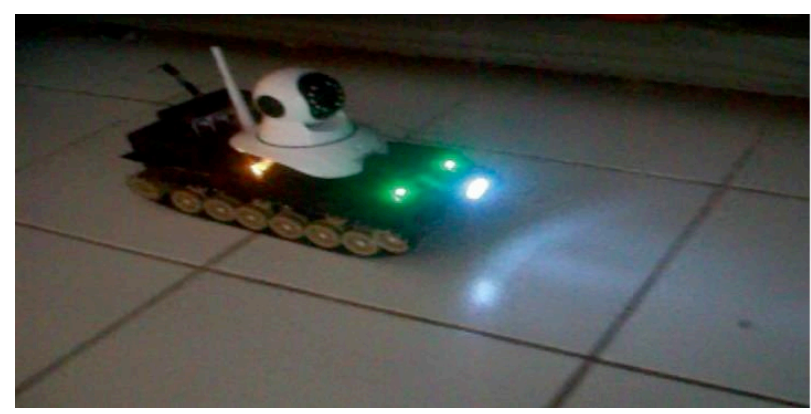

Figura 26: Acionamento do farol.

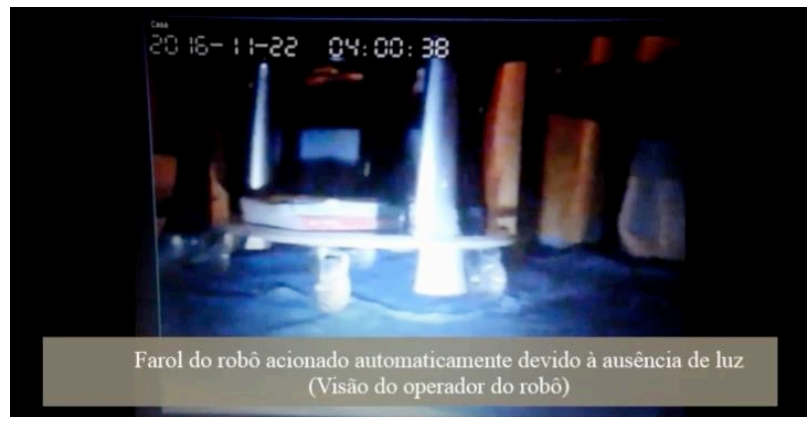

Figura 27: Farol ligado (visão do operador).

\section{Vídeo Demonstrativo}

Realizamos uma filmagem para demonstrar as habilidades do robô e suas características [18].

\section{Conclusões}

Apresento este trabalho sobre o tema, "Plataforma Robótica para busca de Pessoas em locais de risco", abordando a pesquisa e o desenvolvimento de um protótipo robótico, utilizando-se de dois microcontroladores, sensor LDR, módulo de comunicação WI-FI, módulo de controle de motores e um chassi robótico com esteiras.

O protótipo construído e a sua fase de testes mostraram-se inteiramente funcional e bem estável. $O$ desenvolvimento obteve o resultado esperado, e o mesmo pode ser adicionado novas funcionalidades e novos recursos ao longo do seu destino final.

Poderão ser construídas plataformas mais robustas para a idealização do projeto, tornando esta ideia um caminho de abertura para novas maquina com o objetivo de ajudar o ser humano em diversas catástrofes.

A tecnologia usada na sua construção apenas permitirá que ele seja usado em desastre que tenham locais de áreas secas e de temperatura no máximo de $90^{\circ} \mathrm{C}$ e no mínimo de $-40^{\circ} \mathrm{C}$ como já citado poderá ser usado outras tecnologia que permita que o robô atue em locais com temperaturas maiores.

Poderão ser realizados novos testes para a extração das imagens, fazendo com que essas imagens sejam um padrão para reconhecimento de traumas em vitimas de acidentes, usando técnicas de visão computacional.

\section{Referências}

[1] (BRASIL. Constituição (1988). Constituição da República Federativa do Brasil. Brasília, DF: Senado Federal: Centro Gráfico, 1988. 292 p.), Mar 2015.

[2]www.pe.gov.br.http://www.pe.gov.br/orgaos/ corpo-

de-bombeiros-militar-de-pernambuco/, Abr, 2015.

[3] TAN, Chee. http://www.idemployee.id.tue.nl/, 2013. Disponível em: <http://www.idemployee.id. 
tue.nl/g.w.m.rauterberg/publications/AJBAS2013 journal-a.pdf/> Nov, 2016.

[4] S. Sait. startupi.com.br/., 2015. Disponível em: < https://goo.gl/T93GAI />.Abr, 2015.

[5] R. Galileu. Revistagalileu.globo.com., 2010. Disponível em : < https://goo.gl/oLasiH />.Abr, 2015.

[6] Texas A\&M University. crasar.org, 2016. Disponível em : < .http://crasar.org/>.Jun, 2015.

[7] AliExpress. pt.aliexpress.com/, 2015. Disponível em: https://goo.gl/MVnERY / > Mar, 2016.

[8] FRANCETTO, Tiago. tiagofrancetto.blogspot.com.br, 2009.Disponível em: <http://tiagofrancetto.blogspot. com.br/2009/09/tratores-de-esteira.html/>.Abr, 2016.

[9] ANTÔNIO, Marcos. www.pictronics.com.br 2006. Disponível em a. $<$ http://www.pictronics.com.br/ downloads/apostilas/Apostila-Pic-C.pdf/>.Abr, 2016.

[10] SOUZA, Fábio. www.embarcados.com.br, 2014 Disponível em : <http://www.embarcados. com.br/pic/>Abr, 2016.

[11] Blog ProjSeg. blog.ptojseg.com.br. 2014. Disponível em :<http://blog.projseg.com.br/index.php/2014/02 / camera- ip- definicoes funcionalidadesinstalacao-utilizacao/>.Jun, 2016.

[12] Arduino \& Cia. www.arduinoecia.com.br. 2015. Disponível

em:

http://www.arduinoecia.com.

br/2015/03/arduino-modulo-wireless

esp8266.html/ Ago, 2019.

[13] FILIPEFLOP. http://www.filipeflop.com, 2016. Disponível em: $<$ http://www.filipeflop.com/pd -225600-sensorde-luminosidade-Idr-5mm.htm l/> Jun, 2016.

[14] Arduino \& Cia. www.arduinoecia.com.br. 2014. Disponível

em:

http://www.arduinoecia.com .br.http://www.arduinoecia.com.br / 2014 /08 / ponte-h-I298n-motor- de -passo.html.Jun, 2016.
[15] ARIAS, Anderson. blog.droneng.com.br, $2016 . \quad$ Disponível em: $<$ http://blog.droneng.com.br/bateriaslipo/>.Ago, 2016.

[16] LOBO, Júlio. sobrevivencialismo.com, 2016. Disponível em : <https://sobrevivencialismo. com/2016/07/04/ lanternas -o- guia -completo/> Ago, 2016.

[17] DAQUINO, Fernando. www.tecmundo.com.br. 2013 Disponível em: <http://www.tecmundo.com.br/bateria /37110o-que-significa-o-mah-nas-baterias-de-celulares -e-tablets-.htm.Ago, 2016.

[18] NATANAEL, Edgar. www.youtube.com.br, 2016 Disponível em: <https://www.youtube.com/ watch?v=V6ryo8zq36c. Nov, 2016. 